\title{
Direct comparison of CGCRYODERM and DermACELL in the same patient for outcomes in bilateral implant-based breast reconstruction: a retrospective case series
}

\author{
Sungmi Jeon ${ }^{1}$, Jeong Hyun $\mathrm{Ha}^{2}$, Ung Sik Jin ${ }^{1}$ \\ ${ }^{1}$ Department of Plastic and Reconstructive Surgery, Seoul National University Hospital, Seoul, Korea; ${ }^{2}$ Department of Plastic and Reconstructive \\ Surgery, Seoul Metropolitan Government-Seoul National University Boramae Medical Center, Seoul, Korea \\ Contributions: (I) Conception and design: US Jin; (II) Administrative support: US Jin; (III) Provision of study materials or patients: US Jin; (IV) \\ Collection and assembly of data: S Jeon; (V) Data analysis and interpretation: All authors; (VI) Manuscript writing: All authors; (VII) Final approval \\ of manuscript: All authors. \\ Correspondence to: Ung Sik Jin, MD, PhD. Department of Plastic and Reconstructive Surgery, Seoul National University College of Medicine, Seoul \\ National University Hospital, 101 Daehak-ro, Jongno-gu, Seoul 110-744, Korea. Email: usj1011@snu.ac.kr.
}

\begin{abstract}
Background: The use of acellular dermal matrix (ADM) has been popularized in implant-based breast reconstruction (IBR). However, it is still controversial if ADM-associated complication rates differ with varying types of ADM products. The aim of this study was to compare postoperative complications between CGCRYODERM and DermACELL.

Methods: A retrospective chart review was performed on 32 patients (64 breasts) who underwent bilateral prosthetic breast reconstruction between June 2015 and December 2019. All patients received two different $\mathrm{ADMs}$ in each breast during the surgery. Demographic variables, operative characteristics, and postoperative outcomes were compared between the cryopreserved and pre-hydrated ADM.

Results: The overall major and minor postoperative complications developed in 7 and 1 out of 32 patients, respectively. Seroma and infection were the most common complications. There were no cases that infection and/or seroma involved both breasts in one individual. No significant differences were observed in terms of seroma, infection, hematoma, mastectomy flap necrosis, or drainage period between the CGCRYODERM and DermACELL groups ( $\mathrm{P}=0.5637,0.1797,1.0000,0.3173$, and 0.2925 , respectively). There was no case of reconstruction failure leading to explantation.

Conclusions: There were no statistically significant differences in postoperative complications between the two breasts reconstructed with CGCRYODERM and DermACELL in the same patient who underwent bilateral IBR. This is the first study to compare cryopreserved and pre-hydrated ADMs. We suggest that CGCRYODERM is a suitable option with a comparable safety profile for IBR.
\end{abstract}

Keywords: Acellular dermal matrix (ADM); breast reconstruction; breast implants; CGCRYODERM; DermACELL

Submitted Mar 08, 2021. Accepted for publication May 31, 2021.

doi: $10.21037 / g s-21-149$

View this article at: https://dx.doi.org/10.21037/gs-21-149

\footnotetext{
^ ORCID: 0000-0001-9823-0469.
} 


\section{Introduction}

Acellular dermal matrix (ADM) is widely used in implantbased breast reconstruction (IBR). This matrix is a soft connective tissue allograft created by the decellularization process while preserving the intact extracellular skin matrix. When implanted, this structure serves as a scaffold for cell incorporation and revascularization (1). In 2005, Breuing first introduced the use of ADM in IBR for inferolateral pole coverage (2). The introduction of ADM improved aesthetic outcomes, resulting in more natural-looking breasts. In addition, it facilitates tissue expansion in twostage breast reconstruction and offers more opportunities for immediate direct-to-implant (DTI) reconstruction $(3,4)$. Furthermore, IBR using ADM has become a preferred procedure for patients and surgeons, as there is an increasing demand for risk-reducing mastectomy (5).

However, the use of ADM in IBR raises concerns about potential safety issues associated with postoperative complications such as seroma, infection, and explantation (6). As there are various products available in the market, surgeons need more information to reduce $\mathrm{ADM}$-related complications through appropriate surgical techniques, patient selection, and product choice $(7,8)$. ADMs vary significantly depending on the source, processing methods, level of sterility, preparation, biomechanical properties, and available sizes (9). However, it has not been established whether and why there are differences in complication rates among different ADM products (10-13).

Very recently, the U.S. FDA has provided advice on the use of certain brands of ADMs with higher risk profiles in IBR (14). To date, studies comparing the outcomes of different ADMs are relatively limited to specific products such as aseptic freeze-dried Alloderm (LifeCell Corp., Branchburg, NJ, USA), Alloderm Ready-To-Use (RTU), and sterile pre-hydrated DermACELL (LifeNet Health, Virginia Beach, VA, USA) (13,15-18). Further studies are needed to evaluate the outcomes of various types of ADMs.

CGCRYODERM (CGBio Corp., Seongnam, Korea) is an aseptically processed human ADM that was introduced in 2011 as the first using a cryopreservation technique (19). Its manufacturing method can preserve the native dermal matrix structure with adequate tensile strength and abundant growth factors for angiogenesis (20). It requires refrigeration and must be thawed for $3 \mathrm{~min}$, and it has a longer shelf-life than DermACELL stored at room temperature (5 vs. 2 years). On the other hand, freeze-dried
ADM (e.g., Alloderm) requires lengthy rehydration for approximately $30 \mathrm{~min}(21)$.

The objective of our study was to compare the complications between CGCRYODERM and DermACELL in the same patient who underwent bilateral IBR. To the best of our knowledge, this is the first study to compare cryopreserved and pre-hydrated ADM.

We present the following article in accordance with the STROBE reporting checklist (available at https://dx.doi. org/10.21037/gs-21-149).

\section{Methods}

\section{Study population and data collection}

We retrospectively reviewed all patients with unilateral or bilateral breast cancer who underwent bilateral IBR using two different ADMs (CGCRYODERM and DermACELL) at one institute between June 2015 and December 2019. The study was conducted in accordance with the Declaration of Helsinki (revised in 2013). The study was approved by the institutional review board of Seoul National University Hospital (no. 1906-125-104), and individual consent for this retrospective analysis was waived. Patient demographics, operative findings, and postoperative outcomes were collected and analyzed for association with the types of ADMs. Patient outcomes included the following: (I) seroma (subcutaneous fluid collection requiring percutaneous or operative drainage); (II) infection (the presence of a hot, red breast rash requiring additional treatment with antibiotics, surgery, or both); (III) hematoma (requiring surgical exploration); (IV) mastectomy flap necrosis (partial or full-thickness necrosis); (V) capsular contracture [Backer grade III or IV assessed by three plastic surgeons (SMJ, JHH, USJ)]; (VI) prosthesis problems (rupture, deflation, malposition, or exposure); (VII) reconstructive failure (prosthesis explantation as a result of any complication); and (VIII) duration of drainage (postoperative days until all drains were removed). Overall complication rates were analyzed using the outcomes except for duration of drainage, and major complications were separately defined as those requiring unplanned readmission and/or reoperation after discharge for any complication.

\section{Procedures}

Surgical procedures were performed by a single senior plastic surgeon (USJ) at a single institute. In immediate 
reconstruction cases, experienced general surgeons performed total mastectomy (nipple-sparing mastectomy or skin-sparing mastectomy) at the same institute. After confirmation of the frozen biopsy results, reconstruction type (two-stage tissue expander (implant or DTI) was intraoperatively determined depending on the amount of breast skin excised. The prosthetic devices included in this study were of surgeon preference. The TEs used were Biocell $^{\circledR}$-textured Natrelle ${ }^{\circledR} 133$ (Allergan, Inc., Dublin, Ireland) and Siltex ${ }^{\circledR}$-textured MENTOR ${ }^{\circledR} \mathrm{CPX}^{\mathrm{TM}} 4$ (Mentor, Corp., CA, USA). All implants used were MENTOR ${ }^{\circledR}$ CPG $^{\mathrm{TM}}$ (Mentor, Corp., CA, USA) and BellaGel (Hans BioMed, Daejeon, Korea). The author (USJ) used two different ADMs (CGCRYODERM and DermACELL) that were on consignment at the institute during his practice since 2015. In all cases, the ADM was placed in the subpectoral plane as an extension of the pectoralis major muscle. DermACELL is ready to use but should be rinsed briefly in warm saline prior to implantation. Conversely, CGCRYODERM was thawed in warm sterile saline for approximately $3 \mathrm{~min}$. All the drains were placed inferiorly along the inframammary fold. A Jackson-Pratt drain was placed between the ADM and the mastectomy flap while one Hemovac drain was placed between the prosthesis and the ADM. Drains were maintained until the output was less than $30 \mathrm{~mL} /$ day for two consecutive days. All patients received a prophylactic dose of intravenous antibiotics perioperatively during admission to the hospital, and oral antibiotics were continued after discharge (usually 5 days).

\section{Statistical analysis}

All data were queried using Microsoft Excel (Microsoft Corp., Redmond, WA, USA). To evaluate the differences between two breasts from one individual, paired comparison was performed using Wilcoxon's signed rank test for continuous parameters and McNemar's test for parametric parameters. All analyses were performed with IBM SPSS Statistics version 23 (IBM Corp., Armonk, NY, USA). The statistical significance level was set at $\mathrm{P}<0.05$.

\section{Histologic analysis}

After obtaining informed consent, histologic samples were taken from bilateral breast capsules around the ADM in one representative patient at the second stage of breast reconstruction, exchanging TE with permanent implants. Immunohistochemical staining was performed to analyze the expression of alpha-smooth muscle actin ( $\alpha$-SMA) in myofibroblasts and CD31 in endothelial cells. For histological examination, hematoxylin and eosin (H\&E) and Masson's trichrome (MT) stains were used to assess the general (plus cellularity) and collagen structures, respectively.

\section{Results}

Between June 2015 and December 2019, 45 patients underwent bilateral IBR using CGCRYODERM and DermACELL in each breast. Among the 45, 8 patients who had previously undergone breast-conserving surgery, and 5 who underwent different reconstruction methods (one and two stage) for each breast were excluded from this study. The demographics and preoperative characteristics of the 32 patients are shown in Table 1 . The average patient age was 43.9 years, and the mean BMI was $22.9 \mathrm{~kg} / \mathrm{m}^{2}$. There were no smokers or diabetic patients in our study. Ten BRCA1/2 mutation carriers were identified. Among them, one patient was diagnosed with bilateral breast cancer, while 9, who underwent contralateral prophylactic mastectomy, were diagnosed with unilateral breast cancer. Ten of the 32 patients $(31.3 \%)$ received neoadjuvant chemotherapy. Ten patients ( 8 of 38 breasts in the CGCRYODERM group vs. 6 of 38 in the DermACELL group) underwent adjuvant radiation therapy. The mean follow-up period was $925.78 \pm 393.19$ days.

The operative characteristics were summarized in Table 2. Thirty-one of 32 patients $(81.6 \%)$ were reconstructed immediately following bilateral mastectomies, and one underwent bilateral delayed reconstruction with TE following modified radical mastectomy. Sixteen of the 32 breasts $(50 \%)$ were reconstructed with TE (Figure 1), and the remaining underwent DTI (Figure 2). The overall mean mastectomy specimen weight was $360 \pm 195 \mathrm{~g}$. The two groups were comparable in terms of reconstruction method, axillary surgery, size and type of tissue expanders or implants, as well as preoperative characteristics. The mean ADM surface area and mean mastectomy weight showed statistically significant differences in operative characteristics between the CGCRYODERM and DermACELL groups $(\mathrm{P}<0.0001, \mathrm{P}=0.0121$, respectively).

The overall complications in the CGCRYODERM and DermACELL groups were $12.50 \%$ and $15.63 \%$, respectively (Table 3). Major complications requiring readmission and/or reoperation after discharge were not significantly different between the CGCRYODERM and 
Table 1 Overall patient demographics

\begin{tabular}{|c|c|}
\hline Characteristic & Values \\
\hline Number of patients & $\mathrm{N}=32$ \\
\hline Age, years (mean $\pm S D$ ) & $44.25 \pm 8.21$ \\
\hline body mass index, $\mathrm{kg} / \mathrm{m}^{2}$ (mean $\pm \mathrm{SD}$ ) & $22.87 \pm 3.63$ \\
\hline \multicolumn{2}{|l|}{ Smoking status, n (\%) } \\
\hline None & $32(100.00)$ \\
\hline Smokers & $0(0.00)$ \\
\hline \multicolumn{2}{|l|}{ Comorbidities, n (\%) } \\
\hline Diabetes & $0(0.00)$ \\
\hline Hypertension & $2(6.25)$ \\
\hline \multicolumn{2}{|l|}{ Previous breast surgeries, $\mathrm{n}(\%)$} \\
\hline Augmentation mammoplasty & $3(9.38)$ \\
\hline \multicolumn{2}{|l|}{ Laterality, n (\%) } \\
\hline Unilateral breast cancer & $11(34.38)$ \\
\hline Bilateral breast cancer & $21(65.63)$ \\
\hline \multicolumn{2}{|l|}{ Diagnosis, n (\%) } \\
\hline Stage 0 & $7(21.88)$ \\
\hline Stage I & $4(12.50)$ \\
\hline Stage II & $15(46.88)$ \\
\hline Stage III & $6(18.75)$ \\
\hline \multicolumn{2}{|l|}{ BRCA $1 / 2$ mutations, n (\%) } \\
\hline Noncarriers & $22(68.75)$ \\
\hline Carriers & $10(31.25)$ \\
\hline \multicolumn{2}{|l|}{ Chemotherapy, n (\%) } \\
\hline None & $14(43.75)$ \\
\hline Neoadjuvant & $10(31.25)$ \\
\hline Adjuvant & $10(31.25)$ \\
\hline \multicolumn{2}{|l|}{ Radiation therapy, n (\%) } \\
\hline None & $22(68.75)$ \\
\hline Adjuvant & $10(31.25)$ \\
\hline \multicolumn{2}{|l|}{ Hormone therapy, n (\%) } \\
\hline None & $15(46.88)$ \\
\hline Adjuvant & $17(53.13)$ \\
\hline Follow up period, $d$ (mean \pm SD) & $925.78 \pm 393.19$ \\
\hline
\end{tabular}

DermACELL groups (12.50\% vs. $12.50 \%, \mathrm{P}=1.0000)$. All complications occurred within 90 days postoperatively, and it was found that seroma and infection were the most common complications ( 4 of 32 patients). There were no cases that infection and/or seroma involved both breasts in one individual. Only one patient in the DermACELL group underwent implant change in the non-irradiated breast during adjuvant radiation therapy 87 days after surgery. On the other hand, the other patients were treated with intravenous antibiotics and debridement. There were no cases of Baker grade III or IV capsular contracture, any prosthesis problems, or reconstruction failures that led to explantation in our study.

Additionally, there were no significant differences in the drain duration between the CGCRYODERM and DermACELL groups (Table 4).

For histologic analysis, fibrovascular ingrowth (indicating integration within the host tissue at the time of biopsy) and chronic inflammation were observed in both ADMs. There were slightly more spindle cells considered as myofibroblasts in the DermACELL sample than in the CGCRYODERM sample (Figure S1).

\section{Discussion}

This retrospective study demonstrated that there were no significant differences in postoperative complications between the two different human ADMs (CGCRYODERM vs. DermACELL) in bilateral IBR. ADM-related complications are associated with several patient factors which include age, body mass index, smoking status, presence of diabetes, and breast size (6). In addition, reported complication rates vary widely because of different surgical techniques and postoperative management in different centers and different definitions of complications (22). In this study, all reconstructive surgeries were performed by an experienced surgeon. Factors affecting complication risks were reduced by comparing two different ADMs concurrently implanted in the same patient.

This study did not reveal any predictive risk factors for complications associated with ADM-assisted IBR. However, we noted that four patients who developed major infection (one in the CGCRYODERM group and three in the DermACELL group) had several common aspects. All BRCA mutation carriers were diagnosed with unilateral breast cancer. Infection requiring intravenous antibiotics occurred in the contralateral breasts within 90 days after risk-reducing nipple-sparing mastectomy (NSM) and immediate DTI reconstruction. Among four 
Table 2 Operative findings by type of acellular dermal matrix

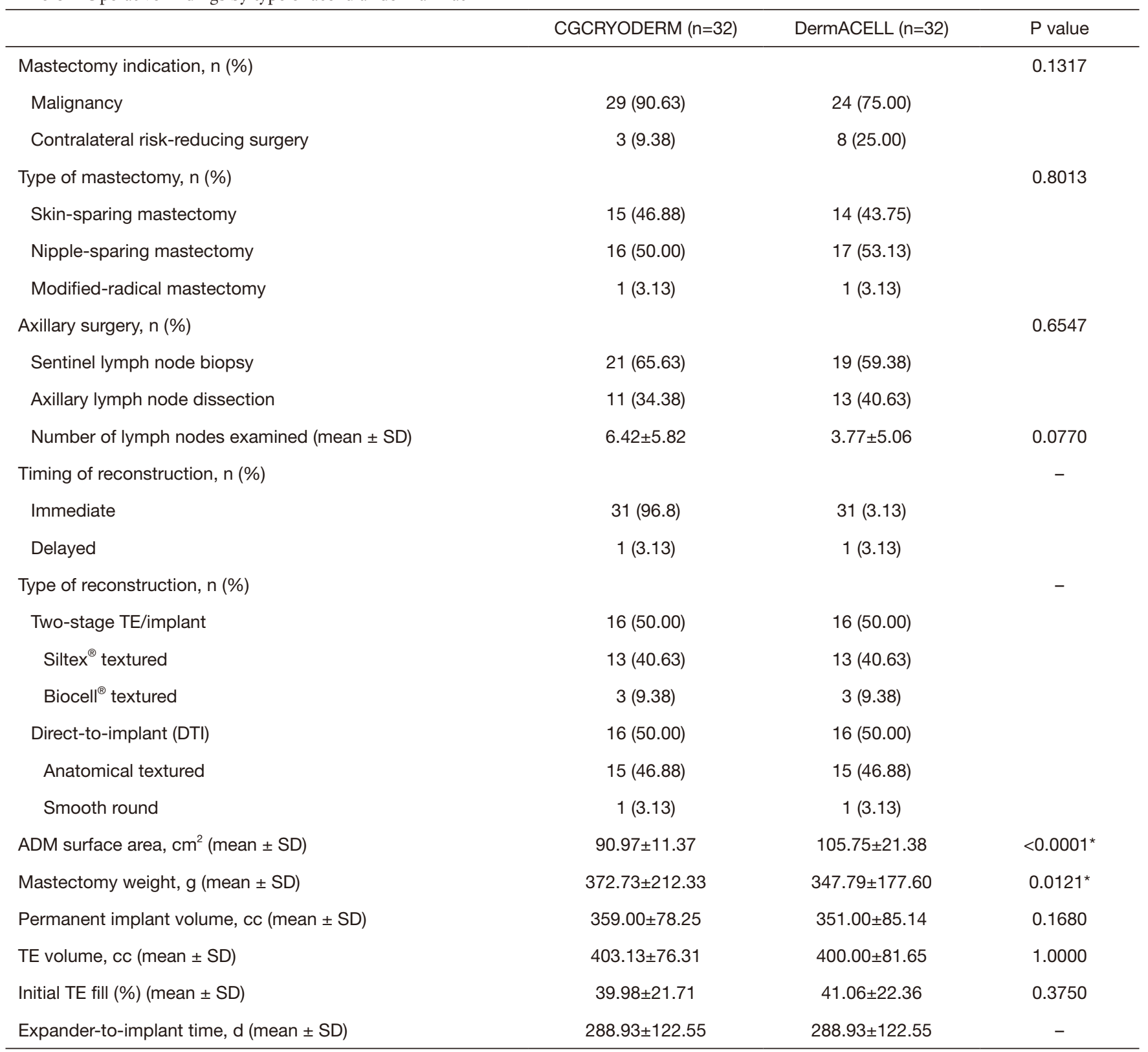

${ }^{*}$, $\mathrm{P}$ value $<0.05$.

patients, three who concurrently developed major seroma (one in the CGCRYODERM group and two in the DermACELL group) received neoadjuvant chemotherapy. In addition, complications developed in non-irradiated breasts before and during radiation therapy.

It is still controversial whether some ADMs are associated with a higher risk of complications than others. Previous studies have shown relatively lower complication rates of sterile pre-hydrated ADMs compared with other aseptic or/and freeze-dried ones $(17,23,24)$. Conversely, there is increasing evidence that no statistically significant differences were observed between sterile versus aseptic $\mathrm{ADMs}$ in drain duration and complications after IBR $(16,18,25,26)$. In this study, the results indicate that CGCRYODERM has a safety profile comparable with that of DermACELL. Moreover, the diversity of available sizes 

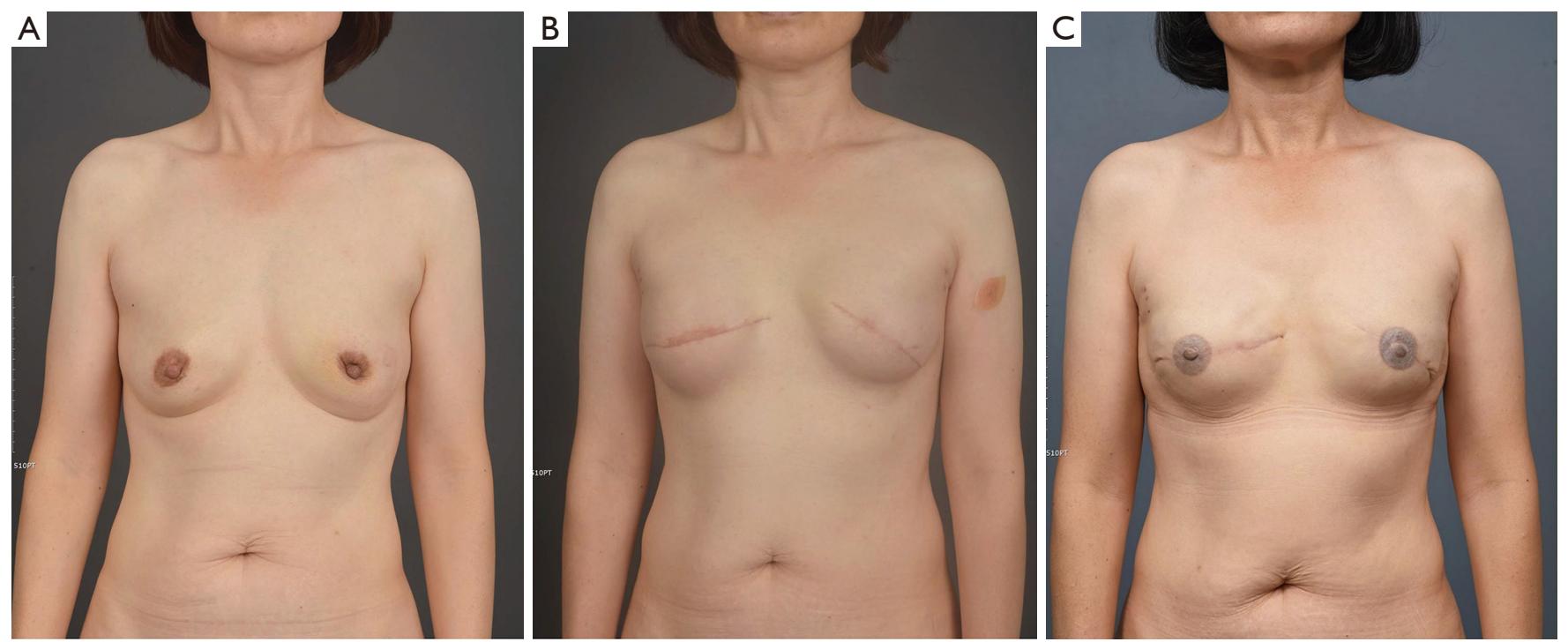

Figure 1 A 51-year-old female patient was diagnosed with bilateral breast cancer (right stage II, left stage 0). The patient underwent immediate two-stage tissue expander/implant reconstruction (right CGCRYODERM $5 \times 16 \mathrm{~cm}^{2}$, left DermACELL $5 \times 16 \mathrm{~cm}^{2}$ ) after bilateral skin-sparing mastectomies (right $268.5 \mathrm{~g}$, left $236 \mathrm{~g}$ ) with sentinel lymph node biopsy. She received adjuvant chemotherapy without complications. (A) Initial photograph, (B) intermediate photograph 7 months after tissue expander insertion (textured tissue expanders Allergan N-67-133FX11 $350 \mathrm{cc}$ each), and (C) postoperative photograph 2 years after the second surgery (textured anatomical implants Mentor CPG321 235cc each).
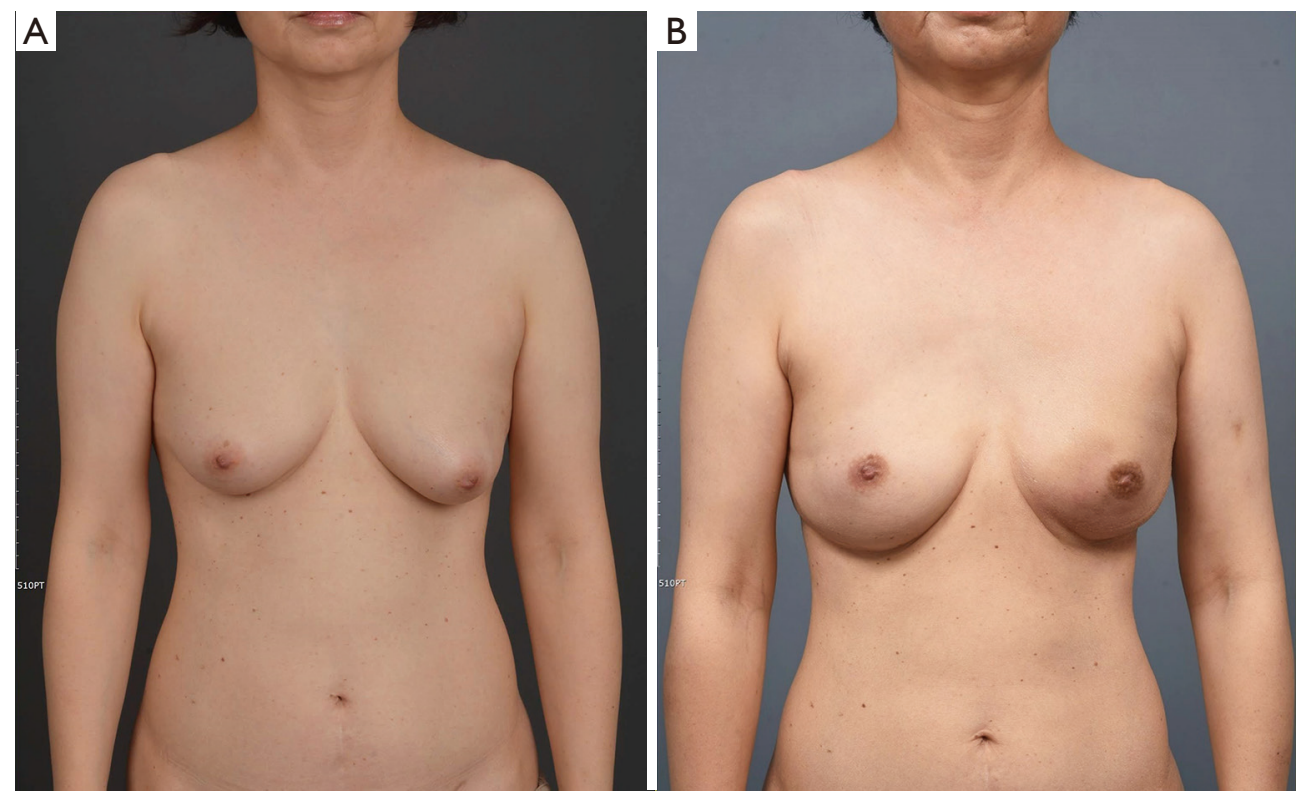

Figure 2 A 51-year-old female patient with BRCA1 mutation. She was diagnosed with left breast cancer (stage II). The patient underwent immediate direct-to-implant reconstruction (right DermACELL $8 \times 16 \mathrm{~cm}^{2}$, left CGCRYODERM $7 \times 15 \mathrm{~cm}^{2}$; textured anatomical breast implants BellaGel BATM-L; right $195 \mathrm{cc}$, left $215 \mathrm{cc}$ ) after bilateral nipple-sparing mastectomies through lateral inframammary fold (right $166 \mathrm{~g}$, left $195.5 \mathrm{~g}$ ) with sentinel lymph node biopsy. She received adjuvant chemotherapy and radiation therapy without complications. (A) Initial photograph, and (B) postoperative photograph 1.5 years after reconstruction. 
Table 3 Postoperative outcomes comparing CGCRYODERM versus DermACELL

\begin{tabular}{|c|c|c|c|}
\hline & CGCRYODERM $(\mathrm{n}=32)$ & DermACELL ( $n=32)$ & $P$ value \\
\hline Major complication, n (\%), [events] & $4(12.50)[5]$ & $4(12.50)[7]$ & 1.0000 \\
\hline Seroma, n (\%) & $1(3.13)$ & $2(6.25)$ & 0.5637 \\
\hline Major ${ }^{\star}, \mathrm{n}(\%)$ & $1(3.13)$ & $2(6.25)$ & 0.5637 \\
\hline Major*, n (\%) & $1(3.13)$ & $3(9.38)$ & 0.3173 \\
\hline Hematoma, n (\%) & $1(3.13)$ & $1(3.13)$ & 1.0000 \\
\hline Major*, n (\%) & $1(3.13)$ & $1(3.13)$ & 1.0000 \\
\hline Mastectomy flap necrosis, $\mathrm{n}(\%)$ & $2(6.25)$ & $1(3.13)$ & 0.3173 \\
\hline
\end{tabular}

${ }^{*}$, requiring unplanned readmission and/or reoperation after discharge for any complication.

Table 4 Duration of drainage comparing CGCRYODERM versus DermACELL

\begin{tabular}{lccc}
\hline Mean duration of drainage \pm SD $(\mathrm{d})$ & CGCRYODERM & DermACELL & P value \\
\hline Subcutaneous (SubQ) & $6.44 \pm 4.16$ & $6.34 \pm 2.75$ & 0.8672 \\
Submuscular (SubM) & $12.50 \pm 3.55$ & $12.28 \pm 4.27$ & 0.2925 \\
\hline
\end{tabular}

may avoid an unnecessarily large surface area associated with additional complications and price (Figure S2).

Different processing, preparation, and sterilization methods of ADMs may impact the histological architecture and subsequent incorporation $(27,28)$. DermACELL is terminally irradiated at a low dose at ultralow temperatures to achieve a sterility assurance level (SAL) of $10^{-6}$ (lower compared with other products with SAL of $10^{-3}$ ). In contrast, CGCRYODERM focused on minimal manipulation to preserve the integrity of the dermal layer structure after a series of decellularization and decontamination. The freezing process, which can irreversibly destroy the matrix structure by ice crystallization, and the drying process, which can further alter the collagen structure by removing bound water surrounding biomolecules, were eliminated $(19,21)$. Theoretically, the more preserved dermal structure of CGCRYODERM may be associated with less inflammatory response (29). Although the histological examination was performed in a single case, the result showed more decreased number of myofibroblasts in the CGCRYODEROM capsule. It is reported that decreased presence of myofibroblasts is related to reduced capsular contracture rates with addition of $\mathrm{ADM}$ in $\operatorname{IBR}(27,30,31)$.

This study had several limitations. First, this retrospective study was performed at a single academic center. Second, the small sample size was insufficient to draw conclusions with high statistical power. Third, there were differences in risk factors between the two breasts in the same patient. Moreover, the laterality of cancer affects the size of the excised breast skin, reconstruction method (one vs. two stages), amount of axillary lymph node dissection, and postoperative radiation. An appropriately powered randomized controlled trial (RCT) is needed to investigate these results further, as in previous RCTs comparing biological and synthetic meshes in the same patient (32-34). Lastly, this study was limited to one representative case for histologic analysis of ADM capsules. Further studies are needed to reveal any significant differences through histological evaluation and the correlation with clinical outcomes.

In conclusion, there was no evidence of inferiority between seroma, infection, hematoma, or mastectomy flap necrosis in CGCRYODERM compared with DermACELL in the same patient who underwent bilateral IBR. Given our 
results, the authors suggest that CGCRYODERM can be used interchangeably with DermACELL in ADM-assisted IBR.

\section{Acknowledgments}

We would like to thank Editage (www.editage.co.kr) for English language editing.

Funding: None.

\section{Footnote}

Reporting Checklist: The authors have completed the STROBE reporting checklist. Available at https://dx.doi. org/10.21037/gs-21-149

Data Sharing Statement: Available at https://dx.doi. org/10.21037/gs-21-149

Peer Review File: Available at https://dx.doi.org/10.21037/ gs-21-149

Conflicts of Interest: All authors have completed the ICMJE uniform disclosure form (available at https://dx.doi. org/10.21037/gs-21-149). The authors have no conflicts of interest to declare.

Ethical Statement: The authors are accountable for all aspects of the work in ensuring that questions related to the accuracy or integrity of any part of the work are appropriately investigated and resolved. The study was conducted in accordance with the Declaration of Helsinki (revised in 2013). The study was approved by the institutional review board of Seoul National University Hospital (no. 1906-125-104), and individual consent for this retrospective analysis was waived.

Open Access Statement: This is an Open Access article distributed in accordance with the Creative Commons Attribution-NonCommercial-NoDerivs 4.0 International License (CC BY-NC-ND 4.0), which permits the noncommercial replication and distribution of the article with the strict proviso that no changes or edits are made and the original work is properly cited (including links to both the formal publication through the relevant DOI and the license). See: https://creativecommons.org/licenses/by-nc$\mathrm{nd} / 4.0 /$.

\section{References}

1. Boháč M, Danišovič L', Koller J, et al. What happens to an acellular dermal matrix after implantation in the human body? A histological and electron microscopic study. Eur J Histochem 2018;62:2873.

2. Breuing KH, Warren SM. Immediate bilateral breast reconstruction with implants and inferolateral AlloDerm slings. Ann Plast Surg 2005;55:232-9.

3. Sbitany H, Langstein HN. Acellular dermal matrix in primary breast reconstruction. Aesthet Surg J 2011;31:30S-7S.

4. Macadam SA, Lennox PA. Acellular dermal matrices: Use in reconstructive and aesthetic breast surgery. Can J Plast Surg 2012;20:75-89.

5. Santosa KB, Oliver JD, Momoh AO. Contralateral prophylactic mastectomy and implications for breast reconstruction. Gland Surg 2021;10:498-506.

6. Sorkin M, Qi J, Kim HM, et al. Acellular Dermal Matrix in Immediate Expander/Implant Breast Reconstruction: A Multicenter Assessment of Risks and Benefits. Plast Reconstr Surg 2017;140:1091-100.

7. Vu MM, Kim JY. Current opinions on indications and algorithms for acellular dermal matrix use in primary prosthetic breast reconstruction. Gland Surg 2015;4:195-203.

8. Lee KT, Hong SH, Jeon BJ, et al. Predictors for Prolonged Drainage following Tissue ExpanderBased Breast Reconstruction. Plast Reconstr Surg 2019;144:9e-17e.

9. Ibrahim AM, Ayeni OA, Hughes KB, et al. Acellular dermal matrices in breast surgery: a comprehensive review. Ann Plast Surg 2013;70:732-8.

10. Nilsen TJ, Dasgupta A, Huang YC, et al. Do Processing Methods Make a Difference in Acellular Dermal Matrix Properties? Aesthet Surg J 2016;36:S7-S22.

11. Lyons DA, Mendenhall SD, Neumeister MW, et al. Aseptic versus Sterile Acellular Dermal Matrices in Breast Reconstruction: An Updated Review. Plast Reconstr Surg Glob Open 2016;4:e823.

12. Hong SE, Kim JH. The relationship of human acellular dermal matrix thickness on complication rate and patientreported outcomes in implant-based immediate breast reconstruction. Gland Surg 2021;10:90-100.

13. Hanson SE, Meaike JD, Selber JC, et al. Aseptic FreezeDried versus Sterile Wet-Packaged Human Cadaveric Acellular Dermal Matrix in Immediate Tissue Expander 
Breast Reconstruction: A Propensity Score Analysis. Plast Reconstr Surg 2018;141:624e-32e.

14. U. S. FDA. Acellular Dermal Matrix (ADM) Products Used in Implant-Based Breast Reconstruction Differ in Complication Rates: FDA Safety Communication. 2021. Available online: https://www.fda.gov/medical-devices/ safety-communications/acellular-dermal-matrix-admproducts-used-implant-based-breast-reconstructiondiffer-complication

15. Bullocks JM. DermACELL: a novel and biocompatible acellular dermal matrix in tissue expander and implant-based breast reconstruction. Eur J Plast Surg 2014;37:529-38.

16. Parikh RP, Brown GM, Sharma K, et al. Immediate Implant-Based Breast Reconstruction with Acellular Dermal Matrix: A Comparison of Sterile and Aseptic AlloDerm in 2039 Consecutive Cases. Plast Reconstr Surg 2018;142:1401-9.

17. Pittman TA, Fan KL, Knapp A, et al. Comparison of Different Acellular Dermal Matrices in Breast Reconstruction: The 50/50 Study. Plast Reconstr Surg 2017;139:521-8.

18. Zenn MR, Salzberg CA. A Direct Comparison of Alloderm-Ready to Use (RTU) and DermACELL in Immediate Breast Implant Reconstruction. Eplasty 2016;16:e23.

19. Kim SY, Lim SY, Mun GH, et al. Evaluating the effectiveness of cryopreserved acellular dermal matrix in immediate expander-based breast reconstruction: a comparison study. Arch Plast Surg 2015;42:316-20.

20. Yoon D, Lee JS, Joo SY, et al. Clinical Outcome of Cryopreserved Acellular Dermal Matrix for Full-Thickness Burns. Macromol Res 2018;26:780-7.

21. Lee JH, Park KR, Kim TG, et al. A Comparative Study of CG CryoDerm and AlloDerm in Direct-to-Implant Immediate Breast Reconstruction. Arch Plast Surg 2013;40:374-9.

22. Clemens MW, Kronowitz SJ. Acellular dermal matrix in irradiated tissue expander/implant-based breast reconstruction: evidence-based review. Plast Reconstr Surg 2012;130:27S-34S.

23. Lewis P, Jewell J, Mattison G, et al. Reducing postoperative infections and red breast syndrome in patients with acellular dermal matrix-based breast reconstruction: the relative roles of product sterility and lower body mass index. Ann Plast Surg 2015;74 Suppl 1:S30-2 .

24. Widmyer AS, Mirhaidari SJ, Wagner DS. Implantbased Breast Reconstruction Outcomes Comparing Freeze-dried Aseptic Alloderm and Sterile Readyto-use Alloderm. Plast Reconstr Surg Glob Open 2019;7:e2530.

25. Arnaout A, Zhang J, Frank S, et al. A Randomized Controlled Trial Comparing Alloderm-RTU with DermACELL in Immediate Subpectoral Implant-Based Breast Reconstruction. Curr Oncol 2020;28:184-95.

26. Klein GM, Singh G, Marquez J, et al. Acellular Dermal Matrix Sterility: Does It Affect Microbial and Clinical Outcomes Following Implantation? Plast Reconstr Surg Glob Open 2019;7:e2355.

27. Yu D, Hanna KR, LeGallo RD, et al. Comparison of Histological Characteristics of Acellular Dermal Matrix Capsules to Surrounding Breast Capsules in Acellular Dermal Matrix-Assisted Breast Reconstruction. Ann Plast Surg 2016;76:485-8.

28. Moyer HR, Hart AM, Yeager J, et al. A Histological Comparison of Two Human Acellular Dermal Matrix Products in Prosthetic-Based Breast Reconstruction. Plast Reconstr Surg Glob Open 2017;5:e1576.

29. Park TH, Chung SW, Song SY, et al. The use of acellular dermal matrix in immediate two-stage prosthetic breast reconstruction provides protection from postmastectomy radiation therapy: a clinicopathologic perspective. J Mater Sci Mater Med 2018;29:27.

30. Tevlin R, Borrelli MR, Irizarry D, et al. Acellular Dermal Matrix Reduces Myofibroblast Presence in the Breast Capsule. Plast Reconstr Surg Glob Open 2019;7:e2213.

31. Kim IK, Park SO, Chang H, et al. Inhibition Mechanism of Acellular Dermal Matrix on Capsule Formation in Expander-Implant Breast Reconstruction After Postmastectomy Radiotherapy. Ann Surg Oncol 2018;25:2279-87.

32. Hansson E, Edvinsson AC, Hallberg H. Drain secretion and seroma formation after immediate breast reconstruction with a biological and a synthetic mesh, respectively: A randomized controlled study. Breast J 2020;26:1756-9.

33. Hansson E, Edvinsson AC, Elander A, et al. First-year complications after immediate breast reconstruction with a biological and a synthetic mesh in the same patient: A randomized controlled study. J Surg Oncol 
2021;123:80-8.

34. Hansson E, Burian P, Hallberg H. Comparison of inflammatory response and synovial metaplasia in immediate breast reconstruction with a synthetic and a biological mesh: a randomized controlled clinical trial. J Plast Surg Hand Surg 2020;54:131-6.
Cite this article as: Jeon S, Ha JH, Jin US. Direct comparison of CGCRYODERM and DermACELL in the same patient for outcomes in bilateral implant-based breast reconstruction: a retrospective case series. Gland Surg 2021;10(7):2113-2122. doi: $10.21037 / g s-21-149$ 

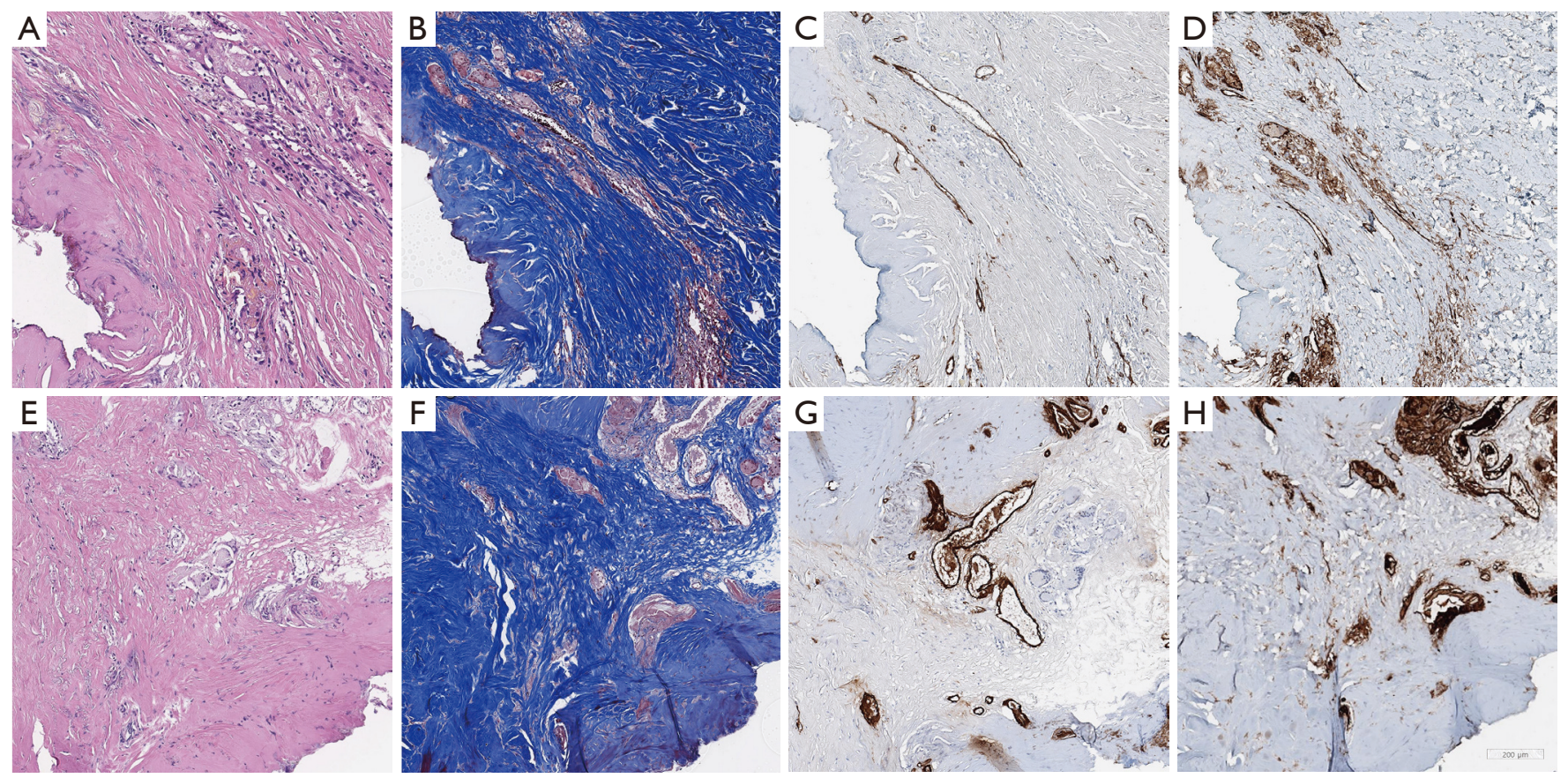

Figure S1 Representative histology images showing the breast capsules around the acellular dermal matrix 8 months after placement. A 39-year-old woman was diagnosed with bilateral breast cancer (right stage II, left stage 0). The patient underwent bilateral two-stage tissue expander/implant reconstruction (textured anatomical implants BellaGel BATM-M; right $340 \mathrm{cc}$, left $310 \mathrm{cc}$ ) after bilateral skin-sparing mastectomies (right $238.5 \mathrm{~g}$, left $243 \mathrm{~g}$ ) with right axillary lymph node dissection and left sentinel lymph node biopsy. The patient received adjuvant hormone therapy without chemotherapy or radiation therapy. (A,B,C,D) CGCRYODERM $6 \times 14$ in the right breast versus $(\mathrm{E}, \mathrm{F}, \mathrm{G}, \mathrm{H})$ DermACELL $5 \times 14$ in the left breast. (A,E) Hematoxylin and eosin (H\&E) staining, (B,F) Masson's trichrome (MT) staining, $(\mathrm{C}, \mathrm{G})$ immunohistochemistry (IHC) for alpha-smooth muscle actin $(\alpha-\mathrm{SMA})$, and $(\mathrm{D}, \mathrm{H})$ IHC for CD31 (magnification $\times 5$, scale bar $200 \mu \mathrm{m})$.

\begin{tabular}{|l|c|c|}
\hline Sizes of ADM $\left(\right.$ surface area, $\left.\mathrm{cm}^{2}\right), \mathrm{n}(\%)$ & CGCRYODERM $(\mathrm{n}=32)$ & DermACELL (n=32) \\
\hline $4 \times 16 \mathrm{~cm}\left(64 \mathrm{~cm}^{2}\right)$ & $\mathrm{N} / \mathrm{A}$ & N/A \\
\hline $5 \times 14 \mathrm{~cm}\left(70 \mathrm{~cm}^{2}\right)$ & $3(9.38)$ & N/A \\
\hline $5 \times 15 \mathrm{~cm}\left(75 \mathrm{~cm}^{2}\right)$ & $3(9.38)$ & $5(15.63)$ \\
\hline $5 \times 16 \mathrm{~cm}\left(80 \mathrm{~cm}^{2}\right)$ & $1(3.13)$ & $1(3.13)$ \\
\hline $6 \times 12 \mathrm{~cm}\left(72 \mathrm{~cm}^{2}\right)$ & $\mathrm{N} / \mathrm{A}$ & $\mathrm{N} / \mathrm{A}$ \\
\hline $6 \times 14 \mathrm{~cm}\left(84 \mathrm{~cm}^{2}\right)$ & $3(9.38)$ & $\mathrm{N} / \mathrm{A}$ \\
\hline $6 \times 15 \mathrm{~cm}\left(90 \mathrm{~cm}^{2}\right)$ & $3(9.38)$ & $11(34.38)$ \\
\hline $6 \times 16 \mathrm{~cm}\left(96 \mathrm{~cm}^{2}\right)$ & $15(46.88)$ & N/A \\
\hline $7 \times 15 \mathrm{~cm}\left(105 \mathrm{~cm}^{2}\right)$ & $2(6.25)$ & N/A \\
\hline $7 \times 16 \mathrm{~cm}\left(112 \mathrm{~cm}^{2}\right)$ & $2(6.25)$ & N/A \\
\hline $8 \times 16 \mathrm{~cm}\left(128 \mathrm{~cm}^{2}\right)$ & 14 (43.75) \\
\hline
\end{tabular}

Figure S2 Sizes of CGCRYODERM versus DermACELL used in this study. 